\title{
Overexpression of an isopentenyl diphosphate isomerase gene to enhance trans-polyisoprene production in Eucommia ulmoides Oliver
}

\author{
Ren Chen', Yoko Harada', Takeshi Bamba², Yoshihisa Nakazawa ${ }^{1 *}$ and Koichiro Gyokusen ${ }^{3}$
}

\begin{abstract}
Background: Natural rubber produced by plants, known as polyisoprene, is the most widely used isoprenoid polymer. Plant polyisoprenes can be classified into two types; cis-polyisoprene and trans-polyisoprene, depending on the type of polymerization of the isoprene unit. More than 2000 species of higher plants produce latex consisting of cis-polyisoprene. Hevea brasiliensis (rubber tree) produces cis-polyisoprene, and is the key source of commercial rubber. In contrast, relatively few plant species produce trans-polyisoprene. Currently, trans-polyisoprene is mainly produced synthetically, and no plant species is used for its commercial production.
\end{abstract}

Results: To develop a plant-based system suitable for large-scale production of trans-polyisoprene, we selected a trans-polyisoprene-producing plant, Eucommia ulmoides Oliver, as the target for genetic transformation. A full-length CDNA (designated as EuIPI, Accession No. AB041629) encoding isopentenyl diphosphate isomerase (IPI) was isolated from E. ulmoides. EulPI consisted of 1028 bp with a 675-bp open reading frame encoding a protein with 224 amino acid residues. EuIPI shared high identity with other plant IPIs, and the recombinant protein expressed in Escherichia coli showed IPI enzymatic activity in vitro. EuIPI was introduced into E. ulmoides via Agrobacterium-mediated transformation. Transgenic lines of E. ulmoides overexpressing EulPI showed increased EuIPI expression (up to 19-fold that of the wild-type) and a 3- to 4-fold increase in the total content of trans-

polyisoprenes, compared with the wild-type (non-transgenic root line) control.

Conclusions: Increasing the expression level of EulPI by overexpression increased accumulation of transpolyisoprenes in transgenic E. ulmoides. IPI catalyzes the conversion of isopentenyl diphosphate to its highly electrophilic isomer, dimethylallyl diphosphate, which is the first step in the biosynthesis of all isoprenoids, including polyisoprene. Our results demonstrated that regulation of IPI expression is a key target for efficient production of trans-polyisoprene in E. ulmoides.

Keywords: Isopentenyl diphosphate isomerase, Trans-polyisoprene, Natural rubber, Genetic transformation, Eucommia ulmoides

\section{Background}

Natural rubber produced by plants, known as polyisoprene, is the most widely used isoprenoid polymer. Plant polyisoprenes can be classified into two types; cis- or $(Z)$-polyisoprene and trans- or $(E)$-polyisoprene, depending on the type of polymerization of the isoprene unit (Figure 1) [1,2]. More than 2000 species of higher plants produce latex consisting of cis-polyisoprene [3]. Hevea

\footnotetext{
* Correspondence: nakazawa@bio.eng.osaka-u.ac.jp

${ }^{1}$ Technical Research Institute, Hitachi Zosen Corporation, Osaka 551-0022, Japan

Full list of author information is available at the end of the article
}

brasiliensis (rubber tree), which produces cis-polyisoprene, is the key source of commercial rubber because of its high rubber yields, and the excellent physical properties of the rubber products [1]. Relatively few plant species produce trans-polyisoprene. Those that are able to produce trans-polyisoprene include Mimusops balata (Balata), Palaquium gutta (Gutta percha) $[4,5]$ and Eucommia ulmoides [6-8]. Trans-polyisoprene has several specific properties that differ from those of cis-polyisoprene. It is more rigid, provides excellent insulation, has an extremely low coefficient of thermal expansion/ contraction, and is resistant to acid and alkali. These

\section{Biomed Central}




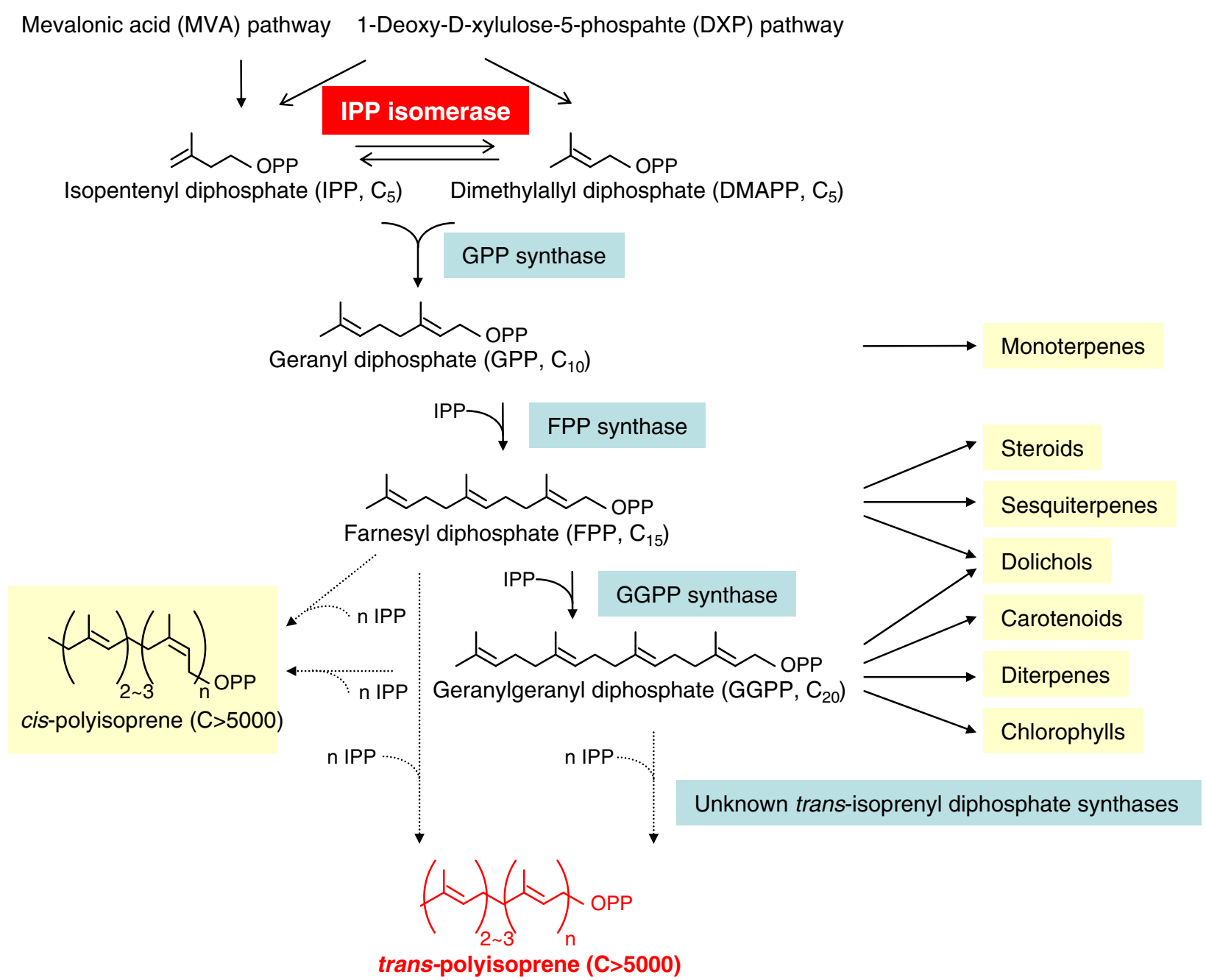

Figure 1 Overview of trans-polyisoprene biosynthesis pathway in plants. In the isoprenoid biosynthetic pathway, the basic five-carbon unit IPP is synthesized in the cytoplasm via the MVA pathway or in plastids via the DXP pathway. Then, IPP is interconverted to its highly electrophilic isomer, DMAPP, by the isomerase IPI at the first step. DMAPP then loses its inorganic pyrophosphate to form isoprene, which sequentially condenses with IPP to generate the short-chain isoprenoid precursors GPP, FPP, and GGPP. These precursors are further metabolized for the biosynthesis of distinct sets of isoprenoids, such as monoterpenes (C10), sesquiterpenes (C15), diterpenes (C20), and polyisoprenes (C>5000) by various isoprenyl diphosphate synthases.

properties could be exploited in the manufacture of insulated cables, moulds, sports goods, and medical or scientific instruments $[9,10]$. Currently, trans-polyisoprene is mainly produced synthetically, and no plantbased systems have been developed for commercial use.

To develop a suitable plant-based system for the largescale production of trans-polyisoprene, we selected a trans-polyisoprene-producing plant, E. ulmoides Oliver, as the target for genetic transformation. E. ulmoides is a deciduous, dioecious woody plant that produces a transpolyisoprene known as Eu-rubber in the leaves, root, bark, and pericarp $[7,8]$. In this study, we isolated and overexpressed an isopentenyl diphosphate isomerase (IPI) gene in E. ulmoides to enhance its trans-polyisoprene production. IPI catalyzes the interconversion of isopentenyl diphosphate (IPP) to its highly electrophilic isomer, dimethylallyl diphosphate (DMAPP), which is an essential starter moiety for the first step in biosynthesis of all isoprenoids including polyisoprene (Figure 1). Previous studies reported that overexpression of the IPI gene caused accumulation of many related downstream isoprenoid metabolites, such as carotenoids and terpenoid indole alkaloids $[11,12]$. All these reports suggested that IPI may be a target enzyme for regulating polyisoprene biosynthesis.

\section{Results}

Cloning and characterization of EuIPI CDNA

To isolate the IPI gene from E. ulmoides, we designed one pair of degenerate primers for RT-PCR based on the 
sequence of a conserved region in the known plant IPIs, including Arabidopsis thaliana IPI (U47324), Bupleurum chinense clone IPI (GQ433719), Camptotheca acuminata IPI1 (AF031079), H. brasiliensis IPI1 (AB294696); Ipomoea sp. Kenyan IPI (AB499048), Nicotiana tabacum IPI1 (AB049815), Periploca sepium IPI (AB091677), Pinus taeda IPI (GQ476784), Solanum lycopersicum IPI (EU253957), and Zea mays IPI (AF330034). The amplified PCR product (228 bp) was used as a probe to screen the cDNA library constructed from a mature E. ulmoides tree. One positive clone carried a full-length cDNA insert that showed the highest homology to known IPIs. This sequence was designated as EuIPI (Accession No. AB041629). Sequence analysis showed that EuIPI was $1028 \mathrm{bp}$ in length, and contained a 675-bp open reading frame (ORF). The ORF encoded a protein of 224 amino acid residues with a predicted molecular mass of 25.96 $\mathrm{kDa}$ and an isoelectric point of 4.68 (Figure 2). Sequence alignment of the deduced amino acid sequence against those of other IPIs revealed that EuIPI showed high identity (84-92.7\%) to other plant IPIs. Similar to other eukaryotic IPIs reported previously, EuIPI contained a number of highly conserved regions that are common to IPIs from higher plants and humans to yeasts [11,13], and two residues, $\mathrm{C}$ (cysteine) and $\mathrm{E}$ (glutamic acid), in the TNTCCSHPL motif and the WGEHEXDY motif
(Figure 2), respectively, which are critical for catalytic activity of the enzyme [13-16].

Phylogenetic analysis based on the comparison of the deduced amino acid sequence of EuIPI with those of other IPIs from different organisms including plants, bacteria, fungi, and animals demonstrated that EuIPI belonged to the plant IPI group (Figure 3a) and had high homology with C. acuminata IPI2 (90.9\% identity, Figure 3b). The phylogenetic analysis suggested that all IPIs evolved from a common ancestor, and that EuIPI shared a common evolutionary origin with other plant IPIs.

\section{Analysis of EulPI enzymatic activity}

To confirm that the gene product EuIPI was a functional IPI, the cDNA was expressed in Escherichia coli BL21 via a pGEX-6P-1 expression system containing a glutathione $\mathrm{S}$-transferase (GST)-tagged fusion protein sequence. This system is suitable for the production of soluble protein in the cytoplasm of $E$. coli. After induction with isopropyl thio- $\beta$-D-galactoside (IPTG), the E. coli cells harboring EuIPI produced a recombinant protein of approximately $56 \mathrm{kDa}$. The digested protein $(\approx 26 \mathrm{kDa})$ was used for the EuIPI activity assay, in which its ability to catalyze the production of DMAPP from IPP was determined by measuring the amount of radioactivity incorporated into acidlabile reaction products (Table 1). The proton nuclear 


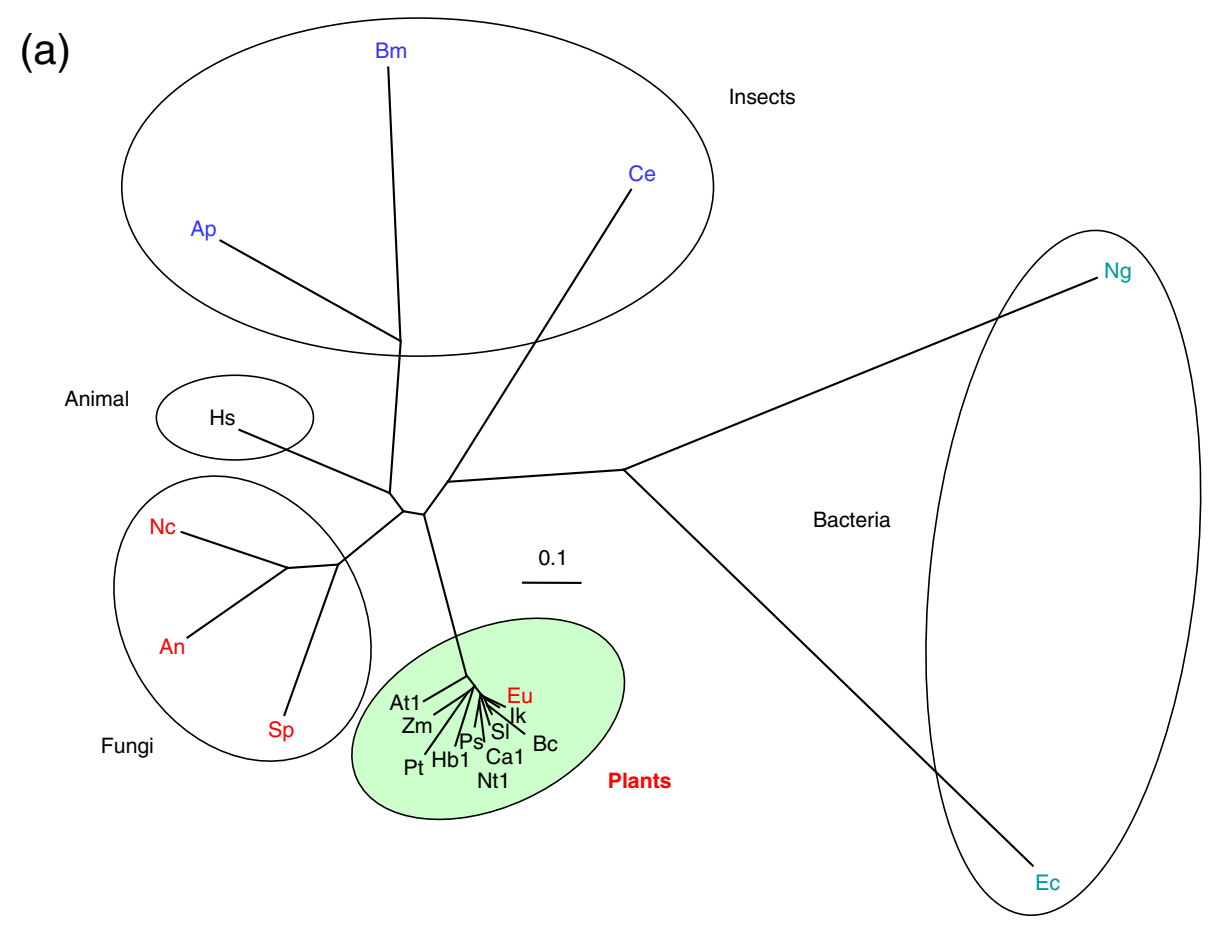

(b)

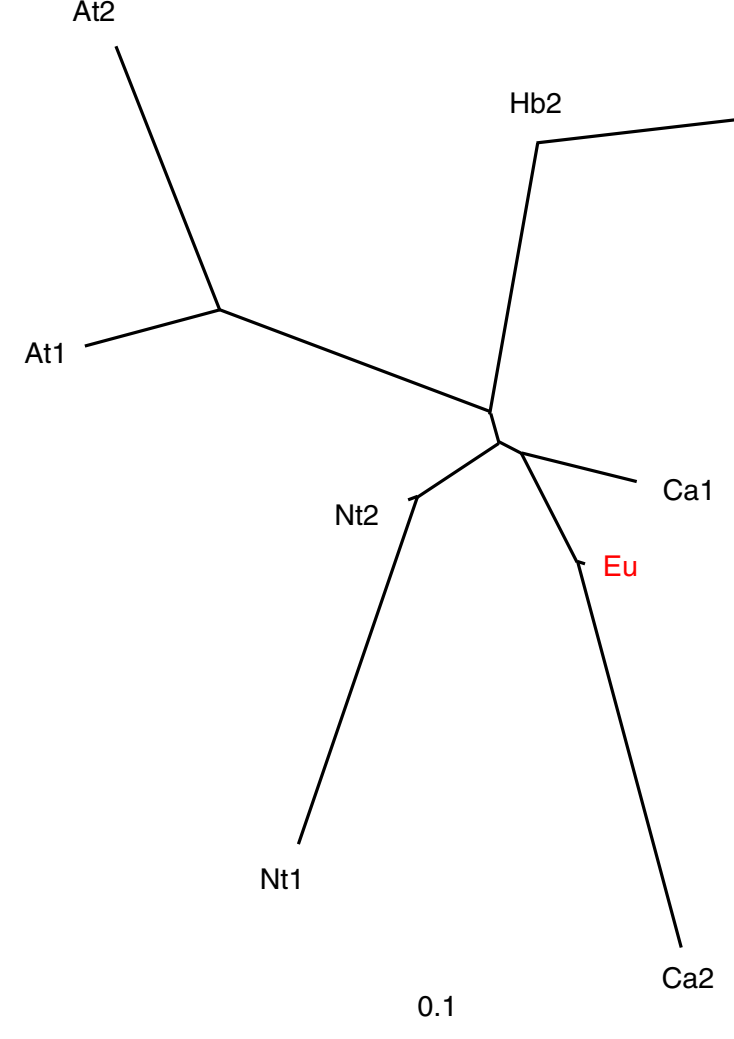

Figure $\mathbf{3}$ (See legend on next page.) 


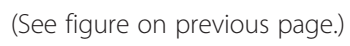

magnetic resonance $\left({ }^{1} \mathrm{H}\right.$ NMR) spectrum from the reaction mixture showed four signals characteristic of DMAPP $(1.53,1.58,4.3$, and 5.3 ppm; Figure $4 \mathrm{a})$, which were absent from the reaction mixture without IPI protein (Figure 4b). The signals of the IPP substrate (1.59, 2.3, 3.9, $4.7 \mathrm{ppm}$; Figure $4 \mathrm{a}, 4 \mathrm{~b})$ were detected in the reaction mixture either with or without purified EuIPI protein. The spectra of IPP and DMAPP were nearly identical to those published previously [17]. These results unambiguously indicated that the digested EuIPI protein was able to catalyze the conversion of IPP to DMAPP in vitro, confirming that the cDNA clone isolated from E. ulmoides encoded a functional IPP isomerase.

\section{Overexpression of EuIPI in E. ulmoides root lines}

The cDNA of EuIPI was inserted into the pMSIsGFP vector, which was introduced into $E$. ulmoides roots via Agrobacterium-mediated transformation. Several kanamycin-resistant root lines with sGFP(S65T) (synthetic green-fluorescent protein with S65T mutation) fluorescence were obtained after selection and regeneration. PCR analysis confirmed that 25 root lines produced the predicted DNA fragment, indicating that the transgenes were present in these transgenic root lines. Some transgenic root lines showed defective phenotypes. Eight PCR-positive $(\mathrm{PCR}+)$ root lines showing strong growth were selected as representative EuIPI-overexpressing transgenic root lines for further analyses.

Real-time RT-PCR analysis of the representative transgenic root lines showed that the expression level of the EUIPI gene was significantly increased in the overexpressing transgenic root lines $(P<0.01$, Figure 5$)$. The highest expression level was in pOEB5-6, which showed 19-fold greater expression than that of the endogenous gene in the wild-type (non-transgenic root line) control. These results confirmed that the overexpression approach successfully upregulated the expression level of EuIPI in transgenic E. ulmoides roots.

\section{Trans-polyisoprene analysis of transgenic E. ulmoides root} lines

The total contents of trans-polyisoprenes in the representative transgenic root lines and their molecular weight distribution were determined by pyrolysis-gas chromatography/mass spectrometry (PyGC/MS) and size exclusion chromatography (SEC). According to the report by Takeno et al. [18], the Soxhlet extraction method coupled with PyGC/MS analysis can remove low-molecular mass biosynthetic intermediates of cisand trans-polyisoprenes, polyprenols, and other isoprenoids such as quinines (plastoquinone or ubiquinone), carotenoids, sterols, and chlorophylls. In addition, polyisoprenes isolated from bark and leaves of E. ulmoides via Soxhlet extraction and fractionation were confirmed to be pure trans-polyisoprene by ${ }^{13} \mathrm{C}$ NMR [7]. Therefore, our PyGC/MS analysis was able to sensitively identify the trans-polyisoprene content in the transgenic lines of E. ulmoides. PyGC/MS analysis of eight representative EuIPI-overexpressing transgenic root lines showed that they contained increased total contents of trans-polyisoprenes (Figure 6), compared with the wildtype control. The trans-polyisoprenes contents in lines pOEB5-3, pOEB5-6, and pOEB5-24 were 3- to 4-fold greater than that in the wild-type control. An overall comparison by analysis of variance (ANOVA) showed that the total contents of trans-polyisoprenes in overexpressing transgenic root lines were significantly greater than those in the wild-type control $(P<0.05)$. These

\section{Table 1 Analysis of EuIPI enzymatic activity}

\begin{tabular}{lcc}
\hline & Background (no enzyme) & EulPI (5 $\mathbf{\mu g})$ \\
\hline$\left[4-{ }^{14} \mathrm{C}\right]$ IPP Incorporation (DPM) & $65.92 \pm 2.86$ & $417.19 \pm 4.86$
\end{tabular}

For EulPI enzymatic activity assay, the $50-\mu \mathrm{L}$ reaction mixture contained $10 \mathrm{nmol}\left[4{ }^{14} \mathrm{C}\right] \mathrm{IPP}(37 \mathrm{GBq} / \mathrm{mol})$ substrate and $5 \mu \mathrm{g}$ purified protein $(0.5 \mu \mathrm{g} / \mu \mathrm{L})$. Radioactivity of reacted products was measured with a liquid scintillation counter as described in the Materials and methods. Distilled water (no enzyme) was used for background measurement; Data represent means \pm standard error, $n=3$. 

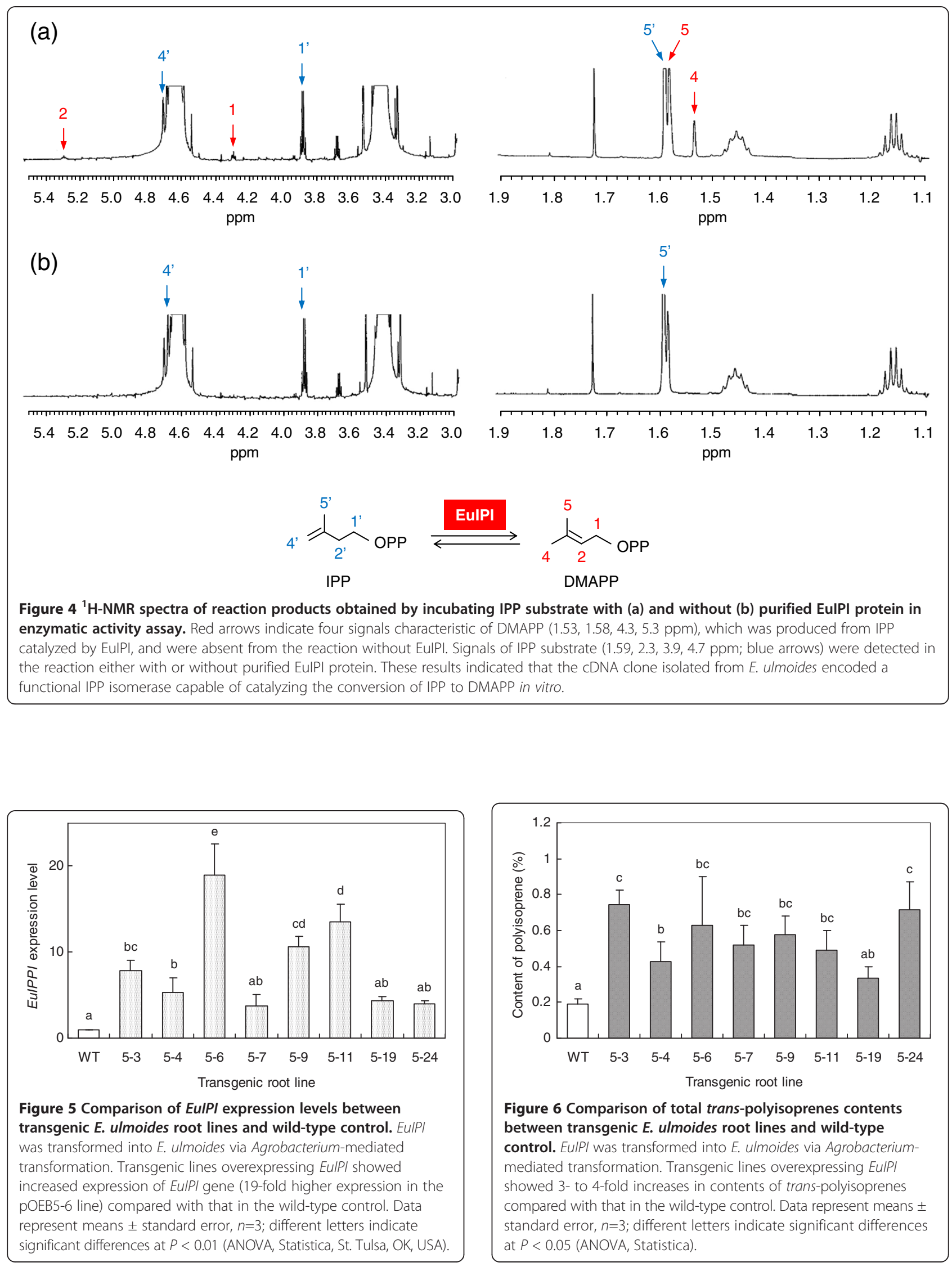
results indicated that up-regulation of EuIPI expression level increased the synthesis of trans-polyisoprenes in $E$. ulmoides root lines.

The molecular-weight distribution of the trans-polyisoprenes extracted from transgenic E. ulmoides root lines and the wild-type control showed a very broad $\left(10^{3}\right.$ to $\left.10^{7}\right)$ and distinct bimodal distribution pattern (Figure 7). The low- and high-molecular weight peaks were centered around $10^{4}$ and $10^{6}$, respectively. Compared with those in the wild-type control, the three representative root lines overexpressing EuIPI (pOEB5-3, pOEB5-6, and pOEB5-24) showed significantly increased peak areas in both the low molar mass region from $1.5 \times 10^{3}$ to $2.5 \times 10^{4}\left(M_{n}=6.3 \times 10^{3}\right.$ and $\left.M_{w}=8.0 \times 10^{3}\right)$ and the high molar mass region from $2.5 \times 10^{4}$ to $4.0 \times 10^{6}$ $\left(M_{n}=3.3 \times 10^{5}\right.$ and $\left.M_{w}=9.3 \times 10^{5}\right)$, especially the latter. This increase was most evident in pOEB5-24. In the transgenic root lines pOEB5-3, pOEB5-6, and pOEB524 , the low molar mass region accounted for 19.2, 16.1, and $13.3 \%$ of all trans-polyisoprenes, respectively, and the high molar mass region accounted for 80.7, 83.9, and $86.7 \%$, respectively. In the wild-type control, the low molar mass region accounted for $26.2 \%$ of all trans-polyisoprenes and the high molar mass region accounted for $73.8 \%$. These results indicated that the transgenic root lines produced higher molecular weight trans-polyisoprenes than did the wild-type control.

\section{Discussion}

We aimed to up-regulate IPI expression to enhance transpolyisoprene production in E. ulmoides. In the plant isoprenoid biosynthetic pathway (Figure 1), the basic five-

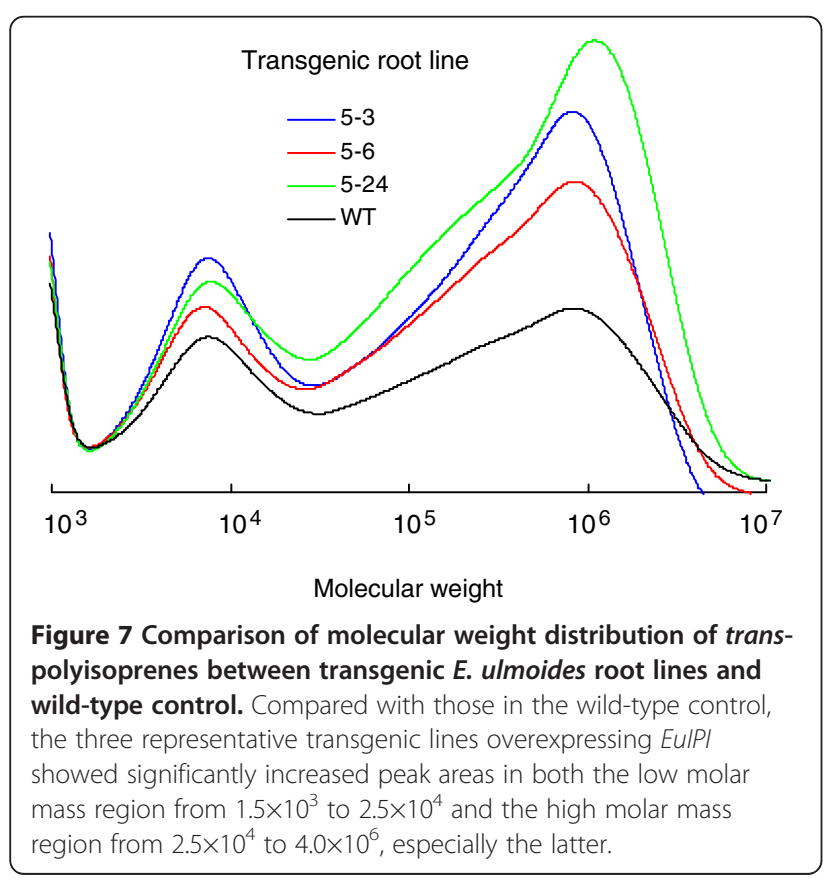

carbon unit IPP is synthesized in the cytoplasm via the mevalonic acid (MVA) pathway $[19,20]$ or in plastids via the 1-deoxy-D-xylulose-5-phosphate (DXP) pathway [9,19-22]. IPP is interconverted to its highly electrophilic isomer, DMAPP, by the enzyme IPI at the first step. Then, IPP is sequentially condensed to DMAPP to yield the short-chain isoprenoid precursors geranyl diphosphate (GPP), farnesyl diphosphate (FPP), and geranylgeranyl diphosphate (GGPP). These precursors are further metabolized for the biosynthesis of distinct sets of isoprenoids, such as monoterpenes (C10), sesquiterpenes (C15), diterpenes $(\mathrm{C} 20)$, and polyisoprenes $(\mathrm{C}>5000)$ by various isoprenyl diphosphate synthases $[20,23,24]$. Therefore, IPP and DMAPP are starting materials at important regulatory branching points in the biosynthetic pathway of a variety of isoprenoids. IPI is thought to catalyze a regulatory step in isoprenoid biosynthesis [20]. It functions in supplying both the electrophilic primer substrate and the condensation substrate for isoprenoid biosynthesis, and provides the precursor for biosyntheses of various meroterpenoids $[11,13,25]$.

According to previous studies, IPIs can be classified into two types: type I and type II. Type I IPIs have been identified in various eukaryotic organisms including humans [26] and Saccharomyces cerevisiae [19], and in some bacteria including E. coli [16] and Rhodobacter capsulatus [27]. The type II enzymes have been identified in archaea and some bacteria. These type II enzymes are FMN and $\mathrm{NAD}(\mathrm{P}) \mathrm{H}$ dependent [17]. To date, no type II IPI has been identified in the plant kingdom. Type I IPIs have a conserved $C$ residue in a TNTCCSHPL motif and a conserved E residue in a WGEHEXDY motif [16]. Type II IPIs have a conserved motif that includes three $G$ (glycine)-rich sequences, MTGG, GXGGT, and (A/G)SGG [17]. These highly conserved residues are critical for the catalytic activity of the enzyme [15,28-30]. Our phylogenetic analysis revealed that EuIPI shared a common evolutionary origin with other plant IPIs. EuIPI also contained the highly conserved $C$ and $E$ residues in the two motifs (Figure 2), suggesting that it belongs to the type I IPI family. The $\mathrm{C}$ and $\mathrm{E}$ residues are thought to face each other in the active site. The reaction is initiated by protonation of the double bond, a process that involves the $\mathrm{E}$ residue. The thiol moiety of $\mathrm{C}$, presumably in the thiolate form, assists in removing the proton from the tertiary cation [29]. Most plants have two type I IPI isozymes with distinct subcellular localizations. In tobacco, IPIs are localized in the cytosol and plastids [31], while in castor bean, IPIs are present in mitochondria and proplastids. Arabidopsis also has two IPI genes that may function in the plastids [20,32,33]. Multiple alignment analysis showed that EuIPI shared high homology with C. acuminata IPI2 (Figure 2), a plastid IPI that resembles other plant IPIs [34]. At present, the subcellular localization of EuIPI is still unknown, and so 
further research is required to clarify its exact location within the cell.

Our results indicated that increasing the expression level of EuIPI increased synthesis of trans-polyisoprene in transgenic E. ulmoides. Overexpression of the IPI gene in $E$. ulmoides enhanced trans-polyisoprene production by 3- to 4-fold compared with that in the wild-type. Although the exact contribution of IPI to biosynthesis of trans-polyisoprene in transgenic E. ulmoides is unknown, our results demonstrated that regulation of IPI expression may be a key target for efficient production of trans-polyisoprene in E. ulmoides. A previous report on the prokaryote $E$. coli implied that maize IPI activity was critical for controlling the flux into the carotenoid pathway [35], and thus, represented an important step in isoprenoid biosynthesis. Similarly, in the green unicellular alga Haematococcus pluvialis, expressions of IPI and two enzymes specific to the carotenoid pathway (lycopene $\beta$-cyclase and $\beta$-carotene-C-4oxygenase) resulted in a 3-to 6-fold increase in carotenoid accumulation after exposure to strong illumination [12]. On the other hand, silencing of the tobacco IPI led to a depletion of photosynthetic pigments, suggesting that reduced IPI activity affected isoprenoid biosynthesis in the plastids of tobacco leaves [36]. A site-directed specific inhibitor of IPI, 3,4-oxido-3-methyl-1-butyl diphosphate (OMBPP), inhibited incorporation of IPP into polyisoprene in an in vitro rubber assay [37]. Together, these findings imply that IPI catalyzes a key step in isoprenoid biosynthesis. Consequently, IPI is an attractive target for metabolic engineering for efficient production of industrially useful isoprenoids, including polyisoprene.

The transgenic root line pOEB5-6 showed the highest expression level of EuIPI, but did not show the highest accumulation of trans-polyisoprene. This may be because EuIPI is a rate-limiting enzyme [11,12,14,25,36] whose catalysis is down-regulated by feedback inhibition [38]. To increase accumulation of trans-polyisoprene via biochemical pathways, it may be necessary to manipulate regulatory genes such as kinases or transcription factors to up-regulate entire pathways [38].

Since a multi-branched metabolic pathway is responsible for the synthesis of distinct isoprenoids, overexpression of only the first-step enzyme, IPI, cannot enhance polyisoprene production to an extremely high level. Recently, we isolated a gene encoding trans-isoprenyl diphosphate synthase (EuTIDS, Accession No. AB041626) and its co-factors, which have roles in prenyl chain elongation and the formation of rubber particle proteins in $E$. ulmoides (data not shown). We anticipate that overexpression of these genes in addition to EuIPI will maximize the production of trans-polyisoprene in E. ulmoides.

E. ulmoides is a tertiary species that survives only in China [39], but it can be cultivated from tropical to temperate zones, and even in cold regions, whereas other species producing trans-polyisoprene (M. balata and $P$. gutta) grow only in the tropics. Hence, $E$. ulmoides shows greater promise as an industrial raw material for commercial use. The results of our study will be helpful to develop E. ulmoides for large-scale production of trans-polyisoprene by genetic engineering.

\section{Conclusions}

To develop a plant-based system suitable for large-scale production of trans-polyisoprene, we selected a transpolyisoprene-producing plant, Eucommia ulmoides Oliver, as the target for genetic transformation. A fulllength cDNA (designated as EuIPI, Accession No. AB041629) encoding isopentenyl diphosphate isomerase (IPI) was isolated from E. ulmoides. EuIPI consists of $1028 \mathrm{bp}$ with a 675-bp open reading frame encoding a protein with 224 amino acid residues. EuIPI shared high identity with other plant IPIs, and the recombinant protein expressed in Escherichia coli showed IPI enzymatic activity in vitro. EuIPI was introduced into E. ulmoides via Agrobacterium-mediated transformation. Transgenic lines of E. ulmoides overexpressing EuIPI showed increased EUIPI expression (up to 19-fold that of the wild-type) and a 3- to 4-fold increase in the total content of trans-polyisoprenes, compared with the wild-type (non-transgenic root line) control. IPI catalyzes the conversion of isopentenyl diphosphate to its highly electrophilic isomer, dimethylallyl diphosphate, which is the first step in the biosynthesis of all isoprenoids, including polyisoprene. Our results demonstrated that regulation of IPI expression is a key target for efficient production of trans-polyisoprene.

\section{Methods \\ cDNA library construction}

Total RNA was extracted from leaves of a mature $E$. ulmoides tree using the cetyltrimethylammonium bromide (CTAB) method [40]. The mRNA was purified from total RNA using Oligotex-dT30 Super (Takara Bio, Otsu, Shiga, Japan) and was used to construct a cDNA library using a lambda ZAP II XR Library Construction kit (Stratagene Japan, Tokyo, Japan).

\section{Cloning of full-length EuIPI cDNA}

The total RNA was reverse-transcribed to synthesize first-strand cDNA using an AMV Reverse Transcriptase XL kit and Oligo dT adaptor primers (Takara Bio). To amplify the EuIPI cDNA fragment, one pair of degenerate primers (forward: $5^{\prime}$ - TTI GTI TGG ACI AAY ACN TGY TG-3' and reverse: $5^{\prime}$-AAA IAG IAG RTA RTC IAN YTC ATG YTC- $3^{\prime}$, where $\mathrm{N}$ is $\mathrm{A}, \mathrm{C}$, G or T; $\mathrm{Y}$ is $\mathrm{C}$ or $\mathrm{T}$; $\mathrm{R}$ is $\mathrm{A}$ or $\mathrm{G}$, and I is inosine) was designed according to a region that is highly conserved among known 
plant IPIs (Figure 3). The PCR conditions were as follows: $5 \mathrm{~min}$ at $94^{\circ} \mathrm{C}$ for preheating, 30 cycles of $1 \mathrm{~min}$ at $94^{\circ} \mathrm{C}$ for denaturation, $1 \mathrm{~min}$ at $54^{\circ} \mathrm{C}$ for annealing, 2 min at $74^{\circ} \mathrm{C}$ for synthesis, and $7 \mathrm{~min}$ at $74^{\circ} \mathrm{C}$ for final extension. The PCR product was labeled using the AlkPhos Direct Labeling and Detection System with CDP-STAR (GE Healthcare Japan, Tokyo, Japan) and used as a probe to screen the cDNA library. Phage plaques were lifted onto a Hybond $\mathrm{N}+$ membrane (GE Healthcare Japan) and hybridized with the labeled probe under the conditions specified by the manufacturer. The positive lambda ZAP II clones were excised in vivo with helper phage to generate subclones in the pBluescript SK(-) phagemid vector (using a lambda ZAP II XR Library Construction kit; Stratagene Japan) and transformed into E. coli SOLR cells (Stratagene Japan) for sequencing. One clone carrying a full-length cDNA insert was chosen and designated as EUIPI.

\section{Phylogenetic analysis}

The deduced amino acid sequence of EuIPI was aligned against those of other IPIs from different organisms, including plants, bacteria, fungi, and animals, by the Clustal W method with default parameters (Slow-Accurate) using Lasergene ${ }^{\circledR}$ MegAlign (DNASTAR, Madison, USA). A phylogenetic tree was constructed by using the neighbor-joining method [41] using Treeview (Glasgow, Scotland, UK).

\section{Analysis of EulPI enzymatic activity}

The EuIPI cDNA, spanning from the start codon (ATG) to the stop codon (TAG) (Figure 2), was amplified by PCR with the following primers: forward: $5^{\prime}$-TAT CTC GAG ATG GGT GAT ACC GCC GTC-3' (XhoI site underlined) and reverse: $5^{\prime}$-TAT GCG GCC GCT AAG CAG ACT GAT TTT C-3' (Not site underlined). The PCR product was inserted into a XhoI- and NotIdigested pGEX-6P-1 vector (GE Healthcare Japan) to yield an expression plasmid with a GST-tagged fusion protein sequence. The plasmid was introduced into $E$. coli BL21. The recombinant proteins were harvested from the $E$. coli transformant cells after being induced by addition of $0.2 \mathrm{mM}$ IPTG. The GST fusion protein was purified by affinity chromatography using a GSTrap FF column (GE Healthcare Japan), and the GST-tag was removed by enzymatic cleavage using PreScission Protease (GE Healthcare Japan). The purified protein was used for the EuIPI activity assay and ${ }^{1} \mathrm{H}$ NMR analysis according to the methods described by Kaneda et al. [17] with some modifications according to the optimum conditions for enzyme activity. For the assay, $10 \mathrm{nmol}$ [4- $\left.{ }^{14} \mathrm{C}\right]$ IPP (37 GBq/mol) (PerkinElmer Japan, Yokohama, Japan) substrate and $5 \mu \mathrm{g}$ purified protein $(0.5 \mu \mathrm{g} /$ $\mu \mathrm{L})$ were incubated in a $50-\mu \mathrm{L}$ reaction mixture containing $100 \mathrm{mM}$ Tris- $\mathrm{HCl}$ (pH 7.0), $5 \mathrm{mM} \mathrm{MgCl}_{2}$, $25 \mathrm{mM} \mathrm{NaF}$, and $1 \mathrm{mM}$ dithiothreitol at $30^{\circ} \mathrm{C}$ for 20 min. The reaction was terminated by adding $200 \mu \mathrm{L}$ methano1: $\mathrm{HCl}(4: 1, \mathrm{v} / \mathrm{v})$ and $500 \mu \mathrm{L}$ water. Lactonization of samples was then carried out at $37^{\circ} \mathrm{C}$ for $10 \mathrm{~min}$. The incubation mixture was saturated with $\mathrm{NaCl}$ and the allylic products were extracted twice with $500 \mu \mathrm{L}$ toluene. The supernatant toluene layer was collected and dried over $\mathrm{Na}_{2} \mathrm{SO}_{4}$. The toluene phase $(500 \mu \mathrm{L})$ was mixed with $3 \mathrm{~mL}$ cocktail and the radioactivity of reacted products was measured with a Tri-Carb 2100 liquid scintillation counter (Packard Instrument, Connecticut, USA).

The purified protein $(1 \mathrm{mg})$ was also reacted with unlabelled 5 mM IPP (Sigma-Aldrich, St. Louis, MO, USA) in a $1649-\mu \mathrm{L}$ reaction mixture containing $100 \mathrm{mM}$ Tris$\mathrm{HCl}$ (pH 8.5), $5 \mathrm{mM} \mathrm{MgCl}_{2}, 25 \mathrm{mM} \mathrm{NaF}$, and $1 \mathrm{mM}$ dithiothreitol at $30^{\circ} \mathrm{C}$ for $16 \mathrm{~h}$. After incubation, the reaction mixture was lyophilized, and the resulting residue was resuspended in $99.9 \% \mathrm{D}_{2} \mathrm{O}$. The reaction products were then analyzed using a $500 \mathrm{MHz}{ }^{1} \mathrm{H}$ NMR spectrometer (Agilent Technologies Japan, Tokyo, Japan). As a control, a reaction mixture that did not contain purified protein was also analyzed.

\section{Construction of plant overexpression vector}

The fragment of EuIPI, spanning from the start codon to the stop codon (Figure 2), was amplified by PCR using the following primers: forward: 5'-TAT CTC GAG ATG GGT GAT ACC GCC GTC-3' (XhoI site underlined) and reverse: $5^{\prime}$-GCT GGT ACC CTA AGC AGA CTG ATT TTC C-3' (KpnI site underlined). The PCR product was directionally inserted into the $\mathrm{XhoI}$ and $K p n \mathrm{I}$ sites of a pMSIsGFP binary vector between a cauliflower mosaic virus (CaMV) 35S promoter and a nopaline synthase (NOS) terminator to construct the overexpression vector pOEB5 (Figure 8). The pMSIsGFP vector was modified from a pMSH1 vector [42] by introducing a cassette containing a $35 \mathrm{~S}-\Omega$ promoter $35 \mathrm{~S}$ promoter with additional omega element translational enhancer [43])-driven $s G F P(S 65 T)$ gene [44] and a NOS terminator from pBIsGFP(S65T) [45] inserted at the HindIII sites. An intron from the castor bean catalase gene CAT-1 was fused within the $\mathrm{N}$-terminal part of the sGFP(S65T) coding sequence to discriminate between Agrobacterium and plant expression (because bacteria cannot splice the intron). The resultant vector pOEB5 was introduced into A. tumefaciens strain LBA4404 [46] using a freeze-thaw transformation method [47].

\section{Overexpression of EuIPI gene in E. ulmoides root lines}

For gene transformation, we used a proliferated root line from a 4-week-old germfree seedling of $E$. ulmoides. The root line was kept in suspension culture in a root proliferation liquid medium containing half-strength 


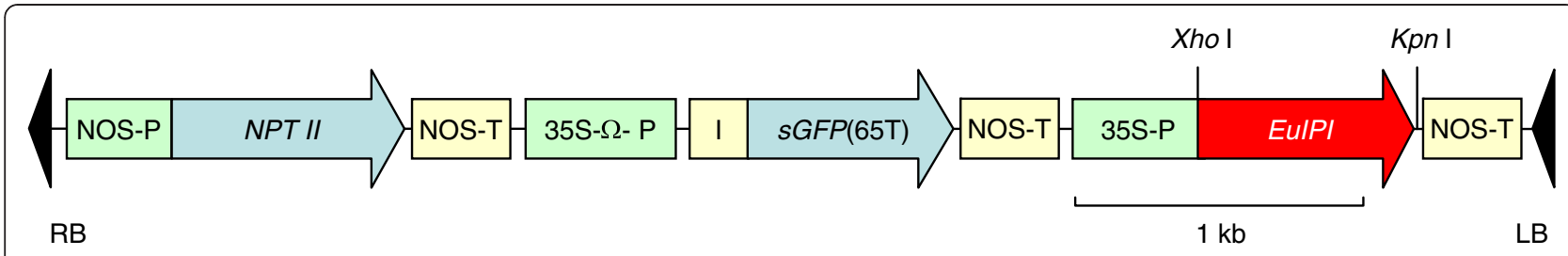

Figure 8 Schematic structure of T-DNA region of overexpression vector used for $E$. ulmoides transformation. CDNA of EulP/ was inserted into pMSIsGFP binary vector between 355 promoter and a NOS terminator. NPT // gene was used as a selective marker, and sGFP(S65T) gene was used to optimize conditions for transformation by monitoring the expression of green-fluorescent protein. An intron was fused within the Nterminal part of the SGFP(S65T) coding sequence to discriminate between Agrobacterium and plant expression (because bacteria can not splice the intron). RB, right border; LB, left border; NOS-P, nopaline synthase promoter; NOS-T, nopaline synthase terminator; 35S-P, cauliflower mosaic virus (CaMV) 35 S promoter; 35S- $\Omega-P$, 35 S promoter with additional omega element translational enhancer; NPT II, neomycin phosphotransferase; sGFP(65T), synthetic green-fluorescent protein with S65T mutation; I: intron of castor bean catalase gene CAT-1.

Murashige and Skoog (MS) basal medium (half-strength MS salts and vitamins), supplemented with $15 \mathrm{~g} / \mathrm{L}$ sucrose and $1 \mu \mathrm{M}$ naphthaleneacetic acid (NAA). The $A$. tumefaciens strain LBA4404 harboring the POEB5 vector was grown overnight at $28^{\circ} \mathrm{C}$ with shaking $(150 \mathrm{rpm})$ in Luria-Bertani liquid medium containing $50 \mathrm{mg} / \mathrm{L}$ kanamycin. Bacterial cells were collected by centrifugation and resuspended to a final $\mathrm{OD}_{550}$ of 0.25 in suspension solution containing MS basal medium supplemented with $30 \mathrm{~g} / \mathrm{L}$ sucrose, $3 \mu \mathrm{M}$ 6-benzylaminopurine (BAP), and $3 \mu \mathrm{M}$ 6-( $\gamma, \gamma$-dimethylallyl-amino)purine (2-iP) combined with $20 \mathrm{mg} / \mathrm{L}$ acetosyringone. The proliferated clonal roots of $E$. ulmoides were cut into 5-8 $\mathrm{mm}$ segments, sonicated for $20 \mathrm{~min}$, and immersed in Agrobacterium suspensions for $3 \mathrm{~min}$. Segments were then blotted dry with sterile filter paper to remove excess bacteria, and transferred into Petri dishes containing filter paper laid over co-cultivation medium (same composition as suspension solution, but solidified with $2.4 \mathrm{~g} / \mathrm{L}$ Gelrite (Wako Pure Chemical Industries, Osaka, Japan). After 3 days of co-cultivation at $22^{\circ} \mathrm{C}$ in the dark, segments were transferred to callus induction and selection medium (same composition as the co-cultivation medium, but with no acetosyringone, and containing $200 \mathrm{mg} / \mathrm{L}$ vancomycin and $25 \mathrm{mg} / \mathrm{L}$ kanamycin). They were subcultured twice over a 3-week interval, and then transferred to root induction medium (same composition as root proliferation liquid medium, but solidified with $2.4 \mathrm{~g} / \mathrm{L}$ Gelrite, and containing $200 \mathrm{mg} / \mathrm{L}$ vancomycin and $25 \mathrm{mg} / \mathrm{L}$ kanamycin). After the calli regenerated adventitious roots, only one well-grown root from each transgenic callus was harvested and transferred to the root proliferation liquid medium containing $200 \mathrm{mg} / \mathrm{L}$ vancomycin and $5 \mathrm{mg} / \mathrm{L}$ kanamycin for proliferation. The roots proliferated from a transgenic callus were considered as an independent root line. All cultures were incubated at $25^{\circ} \mathrm{C}$ under a 16 -h light/8-h dark photoperiod with light supplied by a fluorescent coolwhite light $\left(50 \mu \mathrm{mol} \mathrm{m} \mathrm{m}^{-2} \mathrm{~s}^{-1}\right.$ photosynthetic photon flux density).

\section{DNA and RNA analyses of transgenic roots}

PCR analysis was conducted to screen transgenic roots for the presence of the transgenes. Total genomic DNA was isolated from the regenerated root lines using the DNeasy Plant Mini kit (Qiagen K.K., Tokyo, Japan) according to the manufacturer's instructions. To distinguish the endogenous EuIPI from the transgene, a pair of primers (forward: 5'-TCA TTT GGA GAG AAC ACG GGG GAC-3' and reverse: 5'-TGC TCT TGG ACG TTG CAA ACG TAAG-3') was designed based on the T-DNA sequence of pOEB5 (one from the sequence of the $35 \mathrm{~S}$ promoter and one from that of the NOS terminator, located either side of the EuIPI cDNA overexpression construct). These primers were used to amplify the 820 -bp fragment by PCR using $20 \mathrm{ng}$ total isolated DNA as the template. The PCR conditions were as follows: $5 \mathrm{~min}$ at $95^{\circ} \mathrm{C}$ for preheating, 30 cycles of $1 \mathrm{~min}$ at $95^{\circ} \mathrm{C}$ for denaturation, $1 \mathrm{~min}$ at $60^{\circ} \mathrm{C}$ for annealing, 2 min at $74^{\circ} \mathrm{C}$ for synthesis, and $7 \mathrm{~min}$ at $74^{\circ} \mathrm{C}$ for final extension. Eight PCR+ root lines showing strong growth were selected as the representative EuIPI-overexpressing transgenic root lines for further analyses.

Total RNA was isolated from the eight representative transgenic root lines using an RNeasy Plant Mini kit (Qiagen K. K, Tokyo, Japan). The expression level of EuIPI in these lines was determined by real-time RTPCR as described previously [48]. The primers used for RT-PCR analysis of the EUIPI gene were as follows: $5^{\prime}$ AAC GAT CAG GGA CAA AGG TAA CA- $3^{\prime}$ (forward) and 5'-GGA TGG CTG CAG CAT GTG-3' (reverse). Gene expression was calibrated against that of an endogenous gene, elongation factor-1 alpha $(E F 1 \alpha)$, which was amplified using the primers 5'-CCG AGC GTG AAC GTG GTA T-3' (forward) and $5^{\prime}$-TAG TAC TTG GTG GTT TCG AAT TTC C-3' (reverse).

\section{Analysis of trans-polyisoprenes in transgenic roots}

The total content of trans-polyisoprenes in each transgenic root line and the distribution of their molecular weights (about $10^{2}-10^{8} \mathrm{M}$ ) were determined using 
PyGC/MS and SEC, according to our previous reports [18]. The Soxhlet extraction method was used before PyGC/MS and SEC analyses. Briefly, the sample was lyophilized and ground into a fine powder $(150 \mathrm{mg})$ and then successively extracted by the Soxhlet method with ethanol $(100 \mathrm{~mL})$ at $120^{\circ} \mathrm{C}$ for $10 \mathrm{~h}$ and toluene $(50 \mathrm{~mL})$ at $150^{\circ} \mathrm{C}$ for $12 \mathrm{~h}$. The residue obtained from toluene extraction was rinsed with methanol. The residue was dried with a centrifugal concentrator and then dissolved in toluene. After centrifugation, the supernatant was collected as trans-polyisoprene fractions for PyGC/MS and SEC analyses. For PyGC/MS analysis, polybutadiene rubber (BR, $1 \mathrm{mg}$ ) was added as an internal standard. To quantify trans-polyisoprene, the ratio of the area of trans-polyisoprene to that of BR was compared using NIST MS Search 2.0 (Agilent Technologies, Santa Clara CA, USA). For SEC analysis, a calibration curve was generated using 1,4-polyisoprene standards with SIC480II GPC software (System Instruments, Tokyo, Japan). The molecular weight distribution was bimodal, and the relative areas of low- and high-molecular weight compounds were calculated.

\section{Competing interests}

The authors declare that they have no competing interests.

\section{Authors' contributions}

RC drafted the manuscript, conducted molecular cloning, carried out functional and phylogenetic analyses of EulPI, constructed vectors, analyzed overexpression of EulPI in E. ulmoides, regenerated transgenic plants, and conducted DNA/RNA analyses. YH analyzed trans-polyisoprene in transgenic lines. TB constructed the $\mathrm{CDNA}$ library from E. ulmoides, carried out molecular cloning, analyzed enzymatic activity of EulPI, developed the analysis of trans-polyisoprene in transgenic lines, and helped draft the manuscript. YN conceived the study and participated in its design and coordination. KG participated in the design and coordination of the study. All authors read and approved the final manuscript.

\section{Acknowledgments}

This work was supported by funds from the New Energy and Industrial Technology Development Organization (NEDO), Japan. We thank Prof. Atsuhiko Shinmyo (Nara Institute of Science and Technology) and Dr. Daisuke Shibata (Kazusa DNA Research Institute) for critical reading of the manuscript.

\section{Author details}

'Technical Research Institute, Hitachi Zosen Corporation, Osaka 551-0022, Japan. ${ }^{2}$ Department of Biotechnology, Graduate School of Engineering, Osaka University, Suita, Osaka 565-0871, Japan. ${ }^{3}$ Department of Forest and Forest Products Sciences, Faculty of Agriculture, Kyushu University, Fukuoka 812-8581, Japan.

Received: 16 July 2011 Accepted: 22 October 2012 Published: 30 October 2012

\section{References}

1. Asawatreratanakul K, Zhang YW, Wititsuwannakul D, Wititsuwannakul R, Takahashi S, Rattanapittayaporn A, Koyama T: Molecular cloning, expression and characterization of CDNA encoding cis-prenyltransferases from Hevea brasiliensis. Eur J Biochem 2003, 270:4671-4680.

2. Nakazawa Y, Bamba T, Takeda T, Uefuji H, Harada Y, Li XH, Chen R, Inoue $S$, Tutumi M, Shimizu T, Su YQ, Gyokusen K, Fukusaki E, Kobayashi A: Production of Eucommia-rubber from Eucommia ulmoides Oliv. (hardy rubber tree). Plant Biot 2009, 26:71-79.
3. Backhaus RA: Rubber formation in plants. Israel J Bot 1985, 34:283-293.

4. Archer BL, Audley BG: Rubber gutta percha and chicle. Phytochem 1973, 2:310-343.

5. Schlesinger W, Leeper HM: Chicle - cis- and trans-polyisoprenes from a single plant species. Ind Eng Chem 1951, 43:398-403.

6. Hendricks SB, Wildman SG, Jones EJ: Differentiation of rubber and gutta hydrocarbons in plant materials. Rubber Chem Technol 1946, 19:501-509.

7. Tangpakdee J, Tanaka Y, Shiba K, Kawahara S, Sakurai K, Suzuki Y: Structure and biosynthesis of trans-polyisoprene from Eucommia ulmoides. Phytochem 1997, 45:75-80.

8. Bamba T, Fukusaki E, Nakazawa Y, Kobayashi A: In-situ chemical analyses of trans-polyisoprene by histochemical staining and fourier transform infrared microspectroscopy in a rubber-producing plant, Eucommia ulmoides Oliver. Planta 2002, 215:934-939.

9. Jiang JH, Kai GY, Cao XY, Chen FM, He DN, Liu Q: Molecular cloning of a HMG-CoA reductase gene from Eucommia ulmoides Oliver. Biosci Rep 2006, 26:171-181.

10. Chen R, Namimatsu S, Nakadozono Y, Bamba T, Nakazawa Y, Gyokusen K: Efficient regeneration of Eucommia ulmoides Oliver plant from hypocotyl explant. Biol Plant 2008, 52:713-717.

11. Kajiwara S, Fraser PD, Kondo K, Misawa N: Expression of an exogenous isopentenyl diphosphate isomerase gene enhances isoprenoid biosynthesis in Escherichia coli. Biochem J 1997, 324:421-426.

12. Sun ZR, Cunningham FX, Gantt E: Differential expression of two isopentenyl pyrophosphate isomerases and enhanced carotenoid accumulation in a unicellular chlorophyte. Proc Natl Acad Sci USA 1998, 95:11482-11488

13. Wang YC, Qiu CX, Zhang F, Guo BH, Miao ZQ, Sun XF, Tang KX: Molecular cloning, expression profiling and functional analyses of a cDNA encoding isopentenyl diphosphate isomerase from Gossypium barbadense. Biosci Rep 2009, 29:111-119.

14. Oh SK, Kang H, Shin DH, Yang J, Han KH: Molecular cloning and characterization of a functional cDNA clone encoding isopentenyl diphosphate isomerase from Hevea brasiliensis. J Plant Physiol 2000, 157:549-557.

15. Street IP, Coffman HR, Baker JA, Poulter CD: Identification of Cys 139 and Glu207 as catalytically important groups in the active site of isopentenyl diphosphate: dimethylallyl diphosphate isomerase. Biochem 1994, 33:4212-4217.

16. Hahn FM, Hurlburt AP, Poulter CD: Escherichia coli open reading frame 696 is idi, a nonessential gene encoding isopentenyl diphosphate isomerase. J Bacteriol 1999, 181:4499-4504.

17. Kaneda K, Kuzuyama T, Takagi M, Hayakawa Y, Seto H: An unusual isopentenyl diphosphate isomerase found in the mevalonate pathway gene cluster from Streptomyces sp. strain CL190. Proc Natl Acad Sci USA 2001, 98:932-937.

18. Takeno S, Bamba T, Nakazawa Y, Fukusaki E, Okazawa A, Kobayashi A: Quantification of trans-1,4-polyisoprene in Eucommia ulmoides by fourier transform infrared spectroscopy and pyrolysis-gas chromatography/mass spectrometry. J Biosci Bioeng 2008, 105:355-359.

19. Mayer MP, Hahn FM, Stillman DJ, Poulter CD: Disruption and mapping of IDI1, the gene for isopentenyl diphosphate isomerase in Saccharomyces cerevisiae. Yeast 1992, 8:743-748.

20. Okada K, Kasahara H, Yamaguchi S, Kawaide H, Kamiya Y, Nojiri H, Yamane $\mathrm{H}$ : Genetic evidence for the role of isopentenyl diphosphate isomerases in the mevalonate pathway and plant development in Arabidopsis. Plant Cell Physiol 2008, 49:604-616.

21. Lichtenthaler HK: The 1-deoxy-D-xylulose-5-phosphate pathway of isoprenoid biosynthesis in plants. Annu Rev Plant Physiol Plant Mol Biol 1999, 50:47-65.

22. Rohmer M: The discovery of a mevalonate-independent pathway for isoprenoid biosynthesis in bacteria, algae and higher plants. Nat Prod Rep 1999, 16:565-574.

23. Ogura K, Koyama T: Enzymatic aspects of isoprenoid chain elongation. Chem Rev 1998, 98:1263-1276.

24. Wang $\mathrm{K}$, Ohnuma S: Chain-length determination mechanism of isoprenyl diphosphate synthases and implications for molecular evolution. Trends Biochem Sci 1999, 24:445-451.

25. Ramos-Valdivia AC, van der Heijden R, Verpoorte R: Isopentenyl diphosphate isomerase: a core enzyme in isoprenoid biosynthesis. A review of its biochemistry and function. Nat Prod Rep 1997, 14:591-603. 
26. Xuan JW, Kowalski J, Chambers AF, Denhardt DT: A human promyelocyte mRNA transiently induced by TPA is homologous to yeast IPP isomerase. Genomics 1994, 20:129-131.

27. Hahn FM, Baker JA, Poulter CD: Open reading frame 176 in the photosynthesis gene cluster of Rhodobacter capsulatus encodes idi, a gene for isopentenyl diphosphate isomerase. J Bacteriol 1996, 178:619-624.

28. Durbecq V, Sainz G, Oudjama Y, Clantin B, Gilles CB, Tricot C, Caillet J, Stalon $V$, Droogmans L, Villeret V: Crystal structure of isopentenyl diphosphate: dimethylallyl diphosphate isomerase. EMBO J 2001, 20:1530-1537.

29. Wouters J, Oudjama Y, Barkley SJ, Tricot C, Stalon V, Droogmans L, CD: Catalytic mechanism of Escherichia coli isopentenyl diphosphate isomerase involves Cys-67, Glu-116, and Tyr-104 as suggested by crystal structures of complexes with transition state analogues and irreversible inhibitors. J Biol Biochem 2003, 278:11903-11908.

30. Wouters J, Oudjama Y, Ghosh S, Stalon V, Droogmans L, Oldfield E: Structure and mechanism of action of isopentenylpyrophosphatedimethylallylpyrophosphate isomerase. J Am Chem Soc 2003, 125:3198-3199.

31. Nakamura A, Shimada H, Masuda T, Ohta H, Takamiya K: Two distinct isopentenyl diphosphate isomerases in cytosol and plastid are differentially induced by environmental stresses in tobacco. FEBS Lett 2001, 506:61-64

32. Campbell M, Hahn FN, Poulter CD, Leustek T: Analysis of the isopentenyl diphosphate isomerase gene family from Arabidopsis thaliana. Plant $\mathrm{Mol}$ Biol 1997, 36:323-328.

33. Cunningham FX Jr, Gantt E: Identification of multi-gene families encoding isopentenyl diphosphate isomerase in plants by heterologous complementation in Escherichia coli. Plant Cell Physiol 2000, 41:119-123.

34. Pan XC, Chen M, Liu Y, Wang Q, Zeng LJ, Li LQ, Liao ZH: A new isopentenyl diphosphate isomerase gene from Camptotheca acuminata: Cloning, characterization and functional expression in Escherichia coli. DNA Seq 2008, 19:98-105.

35. Albrecht M, Sandmann G: Light-stimulated carotenoid biosynthesis during transformation of maize etioplasts is regulated by increased activity of isopentenyl pyrophosphate isomerase. Plant Physiol 1994, 105:529-534.

36. Page JE, Hause G, Raschke M, Gao W, Schmidt J, Zenk MH, Kutchan TM: Functional analysis of the final steps of the 1-deoxy-D-xylulose 5phosphate (DXP) pathway to isoprenoids in plants using virus-induced gene silencing. Plant Physiol 2004, 134:1401-1413.

37. Cornish $\mathrm{K}$ : The separate roles of plant cis- and trans-prenyl transferases in cis-1,4-polyisoprene biosynthesis. Eur J Biochem 1993, 218:267-271.

38. Broun P, Somerville C: Progress in plant metabolic engineering. Proc Natl Acad Sci USA 2001, 98:8925-8927

39. Wang JL, Liao XR, Zhang HM, Du JF, Chen PL: Accumulation of chlorogenic acid in cell suspension cultures of Eucommia ulmoides. Plant Cell Tiss Org Cult 2003, 74:193-195.

40. Yamane H, Kashiwa Y, Kakehi E, Yonemori K, Mori H, Hayashi K, Iwamoto K, Tao R, Kataoka I: Differential expression of dehydrin in flower buds of two Japanese apricot cultivars requiring different chilling requirements for bud break. Tree Physiol 2006, 26:1559-1563.

41. Thompson JD, Higgins DG, Gibson TJ, Wolfinger RD: Improving the sensitivity of progressive multiple sequence alignment through sequence weighting, position-specific gap penalties and weight matrix choice. Nucleic Acid Res 1994, 22:4673-4680.

42. Kawasaki T, Henmi K, Ono E, Hatakeyama S, Iwano M, Satoh H, Shimamoto $\mathrm{K}$ : The small GTP-binding protein rac is a regulator of cell death in plants. Proc Natl Acad Sci USA 1999, 96:10922-10926.

43. Benfey PN, Chua NH: The cauliflower mosaic virus $35 \mathrm{~S}$ promoter: combinational regulation of transcription in plants. Science 1990, 250:959-966.

44. Niwa Y, Hirano T, Yoshimoto K, Shimizu M, Kobayashi H: Non-invasive quantitative detection and applications of non-toxic, S65T-type green fluorescent protein in living plants. Plant J 1999, 18:455-463.

45. Kajiyama S, Inoue F, Yoshikawa Y, Shoji T, Fukusaki E, Kobayashi A: Novel plant transformation system by gene-coated gold particle introduction into specific cell using ArF excimer laser. Plant Biotechnol 2007, 24:315-320.

46. Bevan M: Binary Agrobacterium vectors for plant transformation. Nucleic Acids Res 1984, 12:8711-8721
47. Chen H, Nelson RS, Sherwood JL: Enhanced recovery of transformants of Agrobacterium tumefaciens after freeze-thaw transformation and drug selection. Biotechnol 1994, 16:664-670.

48. Chen R, Gyokusen M, Nakazawa Y, Gyokusen K: Selection of housekeeping genes for transgene expression analysis in Eucommia ulmoides Oliver using real-time RT-PCR. J Bot 2010, doi:10.1155/2010/230961.

doi:10.1186/1472-6750-12-78

Cite this article as: Chen et al:: Overexpression of an isopentenyl diphosphate isomerase gene to enhance trans-polyisoprene production in Eucommia ulmoides Oliver. BMC Biotechnology 2012 12:78.

\section{Submit your next manuscript to BioMed Central and take full advantage of:}

- Convenient online submission

- Thorough peer review

- No space constraints or color figure charges

- Immediate publication on acceptance

- Inclusion in PubMed, CAS, Scopus and Google Scholar

- Research which is freely available for redistribution

Submit your manuscript at www.biomedcentral.com/submit
C) Biomed Central 